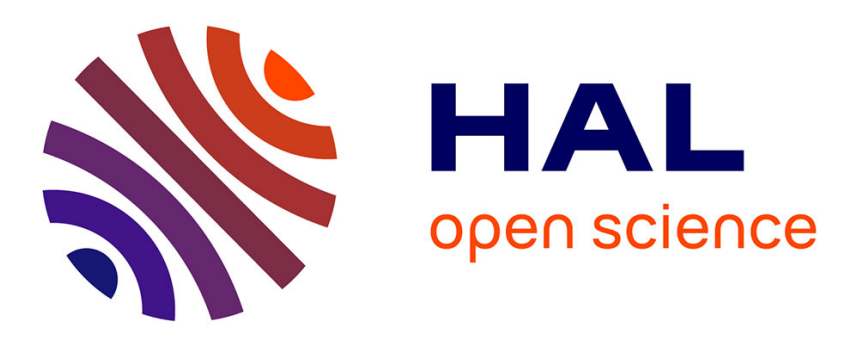

\title{
Bio-Inspired Hovering Control for an Aerial Robot Equipped with a Decoupled Eye and a Rate Gyro
}

\author{
Augustin Manecy, S. Viollet, Nicolas Marchand
}

\section{To cite this version:}

Augustin Manecy, S. Viollet, Nicolas Marchand. Bio-Inspired Hovering Control for an Aerial Robot Equipped with a Decoupled Eye and a Rate Gyro. IROS 2012 - IEEE/RSJ International Conference on Intelligent Robots and Systems, Oct 2012, Vilamoura, Portugal. pp.1110 - 1117, 10.1109/IROS.2012.6385853 . hal-00714332

\section{HAL Id: hal-00714332 \\ https://hal.science/hal-00714332}

Submitted on 24 Apr 2014

HAL is a multi-disciplinary open access archive for the deposit and dissemination of scientific research documents, whether they are published or not. The documents may come from teaching and research institutions in France or abroad, or from public or private research centers.
L'archive ouverte pluridisciplinaire HAL, est destinée au dépôt et à la diffusion de documents scientifiques de niveau recherche, publiés ou non, émanant des établissements d'enseignement et de recherche français ou étrangers, des laboratoires publics ou privés. 


\title{
Bio-Inspired Hovering Control for a Robot Equipped with only a Decoupled Eye and a Rate Gyro
}

\author{
Augustin Manecy, Nicolas Marchand, Stephane Viollet, member IEEE
}

\begin{abstract}
This work provides an hovering control strategy for a sighted robot, the eye of which being decoupled from the body and controlled by means of a tiny rotative piezo motor. The main purpose of this paper is to show the effectiveness and the efficiency of this fundamental bio-inspired mechanical decoupling. Indeed, it exhibits several benefits:

- it enables to stabilize the robot's gaze on the basis of three bio-inspired oculomotor reflexes (ORs) : a visual fixation reflex (VFR), a translational and rotational vestibuloocular reflexes (tVOR and rVOR),

- the eye can better, quickly and accurately compensate for sudden, untoward disturbances caused by the vagaries of the supporting head or body,

- it yields a reference visual signal that can be used to unbias the rate gyro used to implement the VORs and to stabilize the hovering robot,

- it increases the tracking accuracy with moving targets compared to without OR,

This paper shows also that lateral disturbances are rejected 2 times faster with the decoupled eye robot, and roll perturbations induce a retinal error 20 times smaller. The occulomotor reflexes enables to cancel retinal error 6 times faster with 5 times lower retinal error picks. The conclusion of the paper is that decoupled eye must be considered as an efficient autonomous flight solution.
\end{abstract}

\section{ACRONYMS}

$\begin{array}{ll}\text { FOV } & \text { Field Of View. } \\ \text { rVOR } & \text { Rotational Vestibulo-Ocular Reflex. } \\ \text { tVOR } & \text { Translational Vestibulo-Ocular Reflex. } \\ \text { VFR } & \text { Visual Fixation Reflex. } \\ \text { ZSL } & \text { Zero-Setting System. } \\ \text { VFL } & \text { Visual Feedback Loop. } \\ \text { D-EYE } & \text { Decoupled eye system. } \\ \text { F-EYE } & \text { Fixed eye system. }\end{array}$

\section{INTRODUCTION}

In recent years, different techniques were developed to enable UAV's to fly more and more autonomously (automatic taking off and landing, etc.). Most of the proposed strategies are based on a combination of vision sensors and Inertial Measurements Unit. This combination is known to be efficient and is inspired by biological systems like the blowfly which uses both vision and inertial sensing to locate and to estimate its motions. For instance, [1] uses a trajectometry measurement system to get the position and orientation of

A. Manecy is with GIPSA-lab laboratory and ISM laboratory, augustin.manecy@gipsa-lab.fr

N. Marchand is with GIPSA-lab laboratory, Control Systems Dept., SySCo team, CNRS-Univ. of Grenoble, ENSE ${ }^{3}$ BP 46, 38402 St Martin d'Hères Cedex, France nicolas.marchandegipsa-lab.fr

S. Viollet is with the ISM laboratory (Institut of Movement Sciences), Biorobotics Departement stephane.viollet@univ-amu.fr the quad-rotor. This system offers high frequency and good resolution, but is not mobile, and the robot is not totally autonomous. The same idea is exploited by [2] but using a CCD camera. An other strategy is to use markers disposed in the environment (one under the robot and one in front), as in [3] to reconstruct its attitude and position. In the same spirit, the use of embedded camera and different geometrical markers (five) in [4], provides an efficient estimate of both position and orientation of the robot. The same kind of task is too achieved in [5], with markers and but with the coarse visual sensor of the Wii remote. Finally, others technique using optical flow are proposed as in [6] to estimate the altitude, position and speed above a specific geometric road [7]. In all these approaches, the position follows from visual sensors when the attitude is either obtained thanks to the vision as in [1] or with embedded IMU's. In this last case, the gyrometer's bias is classically compensated using the accelerometer.

Contrary to the previously cited papers, in this paper, the robot is assumed to have an "eye" with free movements w.r.t. the "body" of the robot. That way, the visual sensor is able to rotate ant hence to change the gaze (eye's line of sight). This additional degree of freedom mimicks the mechanical decoupling between eye and body that is so characteristic of animals such as for instance the hoverfly. However, we will consider the robots eye as a sensitive and accurate visual position-sensing device (PSD), able to sense the position of an edge (or a bar) within only its small field of view FOV (here, FOV $=5$ in opposition to FOV upper than 50 in the previous references). This sensors performance in the task consisting of locating an edge is a 40 -fold improvement in resolution versus the interphotodiode angular resolution [43]. It can, therefore, be said to be endowed with hyperacuity [44]. For further details about the performance (i.e., accuracy and calibration) of this hyperacute visual PSD, see [40] and [43]. Gaze stabilization is a difficult task because the eye control system must compensate both quickly and accurately for any sudden, untoward disturbances caused by the vagaries of the supporting head or body. This finely adapted mechanism is way beyond what can be achieved in the field of present-day robotics. In addition with this controlled visual sensor, we assume that only a rate-gyro with an unknown bias embarked on the robot. This is more restrictive w.r.t. other contributions where an entire IMU is used.

The aim of this paper is to emphasize the important advantages of this added degree of freedom, even in the case when less MEMS sensors are embarked. For this, a robot with a decoupled eye is presented in the next section as well 
as its nonlinear dynamical model. In Section III, an observer is proposed to estimate position, speed, angles and rate-gyro bias. Based on this observer, the eye control and then the whole robot control are given. Section IV gives an extensive comparison of the behaviour of the robot with a decoupled eye and without. These results are then discussed in the last section.

\section{SYSTEM OVERVIEW}

\section{A. Description of the twin-engine hovering robot}

As shown in the CAD of figure 1, the robot will consist of a twin-engine aerial robot with three degrees of freedom (rotation around the horizontal axis: $\theta_{r}$, right and left translation: $X$ and an eye rotation $\theta_{e r}$ with respect to the robot's body). Thanks to a mechanical decoupling between the eye and its mechanical support (the head), the eye can rotate freely in the robot's frame. In addition, the eye's orientation could be finely controlled by means of a fast and accurate piezo motor (PCB motor) featuring an extreme compactness (diameter of $20 \mathrm{~mm}$ ) and a very low mass (1 gramm). The mass of the overall robot will be about 100 grams and the robot will be completely autonomous in terms embedded computational resource and power supply. A wireless link based on a Bluetooth module will be implemented for the monitoring of several parameters and the sending of input reference signals to the robot from a personal computer.

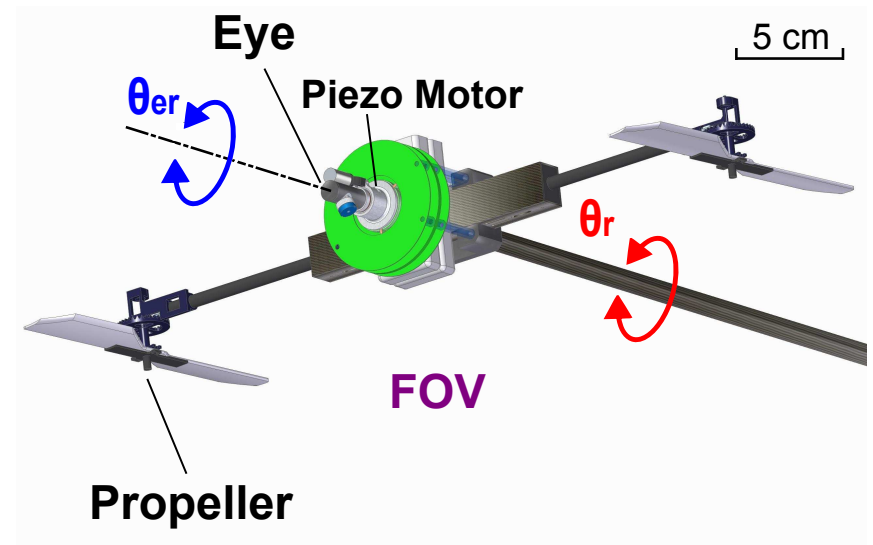

Fig. 1. CAD view of the 100-grams micro-air vehicle in which the miniature rotary piezomotor (PCB Motor) controls the orientation of the eye relative to the body 'angle $\theta_{e r}$ ). The orientation of the robot around the roll axis (angle $\theta_{r}$ ) is controlled by applying a differential rotation speed onto the propellers. The robot itself is mounted at the tip of a rotating arm allowing an horizontal translation of the robot.

As shown in figure 3, the UAV roll angle $\theta_{r}$ can be controlled by applying a differential rotational speed on the propellers. We assumed in this work that the robot flies at a constant altitude $H$. As the flying robot is under-actuated, its position $X$ along the horizontal axis was controlled by adjusting its attitude around the roll axis. As a consequence, the robot remained still in space (hovering condition) when $\theta_{r}=0^{\circ}$. In this work, the robot was considered to hover above a target placed onto the ground.

In this paper, we deliberately placed our hovering control strategy in a bio-inspired minimalistic framework where the objective was to stabilize an underactuated hovering robot by means of only a drifting rate-gyro and an eye with a limited field-of-view. Figure 2 shows that the future realization of our robot will share many similarities with the fly :

- A rate-gyro : the fly has gyroscopic haltere organ measuring its bodys angular speed around the three degree of rotation (pitch, roll and yaw) (citer Hengstenber) whereas the robot is equipped with a classical MEMS rate-gyro.

- An optical position sensing device : the compound eye of the fly is able to locate a contrasting target placed in a small fontal part of the visual field (citer Collett and Boedecker) and the robot is equipped with an eye endowed with hyperacuity (citer Vodka).

- a neck : the fly has non-less than 23 pairs of muscles to control its heads orientation (citer Straussfeld). The robot has a decoupled eye actuated by means of a tiny position servomotor (rotative piezo motor).

- A proprioceptive sensor in the neck : the fly has prosternal organs which consists of a pair of mechanosensitive hair fields located in the neck region (citer Preuss Hengstenberg) and the robot is equipped with a contactless magnetoresistive sensor measuring the orientation of the eye relative to the head.

- A gaze stabilization : in the freely flying sandwasp, active gaze-stabilization mechanisms prevent the incoming visual information from being affected by disturbances, such as large bodys rotations around the roll axis (Citer Zeil).

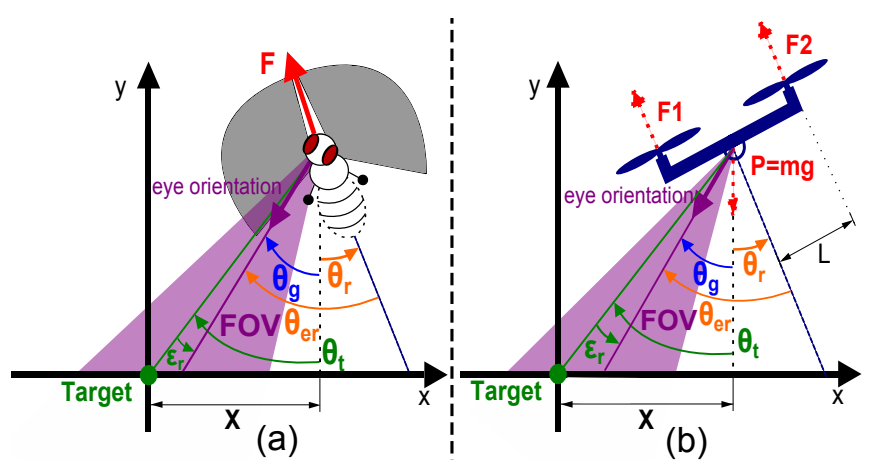

Fig. 2. Similarities between the hovering robot with a decoupled eye (b) and a fly (a). These two dynamic under-actuated systems are able to measure their bodys rotational speed $\Omega_{r}$ by means of a rate-gyro (for the robot) and halteres (for the fly) and to locate a contrasting target $\theta_{t}$ placed in a small part of their FOV. The fly has non-less than 23 pairs of muscles in its neck to stabilize its gaze $\theta_{g}$ whereas the robot can control the angular position of its eye $\theta_{e} r$ by means of miniature rotative piezo motor.

To facilitate the following considerations, we introduced some notation.

- $\theta_{t}$ : angular position of the target in the inertial frame.

- $\theta_{r}$ : roll angle of the robot.

- $\theta_{e r}$ : angle between the eye and the robot in the robot frame. This angle is mechanically constrained to a maximum angle: $\left|\theta_{\text {er }}\right|<\theta_{\text {er_M }} A X$. 
- $\theta_{g}$ : angular position of the gaze in the inertial frame $\theta_{g}=\theta_{e r}+\theta_{r}$.

- $\epsilon_{r}$ : retinal error defined by $\epsilon_{r}=\theta_{t}-\theta_{g}$.

- $X$ : position of the robot along the horizontal axis in the inertial frame.

- $V_{x}$ : speed of the robot along horizontal axis in the inertial frame.

- $Y$ : position of the robot along the vertical axis in the inertial frame. $Y$ was supposed to be constant $(Y(t)=$ $H \forall t$ ). This assumption is not too strong because the control of height could be decoupled of the other control (citer ...) and realized independently.

- $\Omega_{r}$ : roll rotational speed of the robot.

Estimated values are denoted by an additional hat (e.g., $\hat{\theta})$. Reference values are denoted with a star (e.g., $\left.\theta^{\star}\right)$ and measured values are denoted with a bar (e.g., $\bar{\theta}$ )

\section{B. Non-linear model of the hovering robot}

A classical non-linear dynamic model was assumed for the robot in the inertial frame:

$$
\left\{\begin{aligned}
\dot{V}_{x} & =\frac{-\left(F_{1}+F_{2}\right) \sin \left(\theta_{r}\right)}{m}-K_{v x} V_{x} \\
\dot{X} & =V_{x} \\
\dot{\Omega}_{r} & =\frac{L\left(F_{2}-F_{1}\right)}{I_{z}} \\
\dot{\theta}_{r} & =\Omega_{r}
\end{aligned}\right.
$$

Where $\mathrm{L}$ is the distance between the robots center and a propeller, $I_{z}$ is the inertia momentum around the roll axis, $F_{1}$ and $F_{2}$ are respectively the thrust generated by the propeller 1 and 2 and $K_{v x}$ is the flapping coefficient which is supposed to be constant.

An inner speed feedback loop makes the robots angular speed $\left(\Omega_{r}\right)$ follow faithfully the rotational speed set points $\left(\Omega_{r}^{\star}\right)$ yielded by the attitude controller (see III-E). To control the rotational speed $\left(\Omega_{r}\right)$, we assumed that the propellers are controlled directly by adjusting the thrust value. Therefore the control input signal for a propeller is composed of a nominal thrust and a differential thrust. The nominal thrust $\left(T^{\star}\right)$ counteracts the gravity and the differential thrust $\left(\delta^{\star}\right)$ generates the torque responsible of a roll rotation. The propeller control input signals are defined by $F_{1}^{\star}(p)=T^{\star}(p)-\delta^{\star}(p)$ and $F_{2}^{\star}(p)=T^{\star}(p)+\delta^{\star}(p)$. We consider the dynamic of the propellers as a first order system with a time constant equal to $\tau_{m o t}$ (see table ??).

Figure 3 shows the complete model of the robot including the model of the propellers and the model of the rate-gyro, the visual sensor and the angle sensor (used for measuring the eye in robot angle $\theta_{e r}$ ). For the dynamic simulations of the robot, computed under Matlab/Simulink environment, we used a set of parameters given in table I (see Appendix).

\section{HOVERING BY GAZING}

This work aims at describing a new control strategy for implementing a robust, accurate and fast hovering flight. In our approach where the gaze control takes a key role
([8] and [9]), the "hovering-by-gazing", which is presented here, amounts to maintaining the gaze automatically oriented toward a stationary (or moving) target. Contrary to many previous approaches (citer...) where the robots attitude is estimated from an inertial measurement unit (IMU) including rate gyro, accelerometers and magnetormeters, the robots roll $\theta_{r}$ and its position $X$ (with respect to the target) are here estimated only from the robots angular speed $\Omega_{r}$ and the eye-in-robot orientation $\theta_{e r}$. Our control strategy makes the robot minimize its retinal error signal thanks to different occulomotor reflexes and hover accurately above a target by estimating its attitude and position. Here, we will describe the eye control system and the hovering control system and explain how they interact.

\section{A. The non-linear observer}

As shown in figure 4, the state observer is the corner stone of our hovering control strategy. This observer delivers at its output non-less than 4 estimated states on the basis of only two measurements: the eye-in-robot orientation $\bar{\theta}_{e r}$ and the robots angular speed $\bar{\Omega}_{r}$. Indeed, it provides an estimation of linear speed $V_{x}$, position $X$, roll angle $\theta_{r}$ and rate-gyro bias $\Delta_{g}$ :

$$
\left\{\begin{array}{c}
\left(\begin{array}{c}
\dot{\hat{V}}_{x} \\
\dot{\hat{X}} \\
\dot{\hat{\theta}}_{r} \\
\hat{\hat{\Delta}}_{g}
\end{array}\right)=\left(\begin{array}{c}
-\frac{T}{m} \sin \left(\hat{\theta}_{r}\right)-K_{v x} \hat{V}_{x}+L_{1}\left(\bar{\theta}_{e r}-\hat{\theta}_{e r}\right) \\
\hat{V}_{x}+L_{2}\left(\bar{\theta}_{e r}-\hat{\theta}_{e r}\right) \\
\bar{\Omega}_{r}-\hat{\Delta}_{g}+L_{3}\left(\bar{\theta}_{e r}-\hat{\theta}_{e r}\right) \\
L_{4}\left(\bar{\theta}_{e r}-\hat{\theta}_{e r}\right)
\end{array}\right) \\
\hat{\theta}_{e r}=-\arctan \left(\frac{\hat{X}}{Y_{0}}\right)-\hat{\theta}_{r}
\end{array}\right.
$$

We decided to implement a non linear observer because of the strongly non linear equations giving the evolution of the linear speed $V_{x}$ and the position $X$ of the robot (see equation (2)). Non linearities in $\hat{V}_{x}$ provide better estimation during transient, and non linearities in $\hat{\theta}_{e r}$ steady state error in position estimation. Observer gain $L=\left(\begin{array}{llll}L_{1} & L_{2} & L_{3} & L_{4}\end{array}\right)^{T}$ was tuned using the classical LQG method using the linearised system around the origin.

\section{B. An unbiased rate-gyro}

To increase the efficiency of the observer, the rate-gyro bias is estimated, assuming that this bias is slowly varying. But, instead of using a classical unbias method based on the accelerometer measurements, we choose to estimate the rate-gyro bias only thanks to the eye system. And more particularly, thanks to the eye-in-robot angle which provides us a good means to avoid drift in position estimation. As a consequence, the rate-gyro bias is observable, only if the position estimation is accurate. This accuracy is achieved thanks to the non-linearity of the estimated output $\hat{\theta}_{e} r$.

$$
\begin{aligned}
& \bar{\Omega}_{r}=\Omega_{r}+\Delta_{g}+\mu \\
& \dot{\Delta}_{g}=0
\end{aligned}
$$




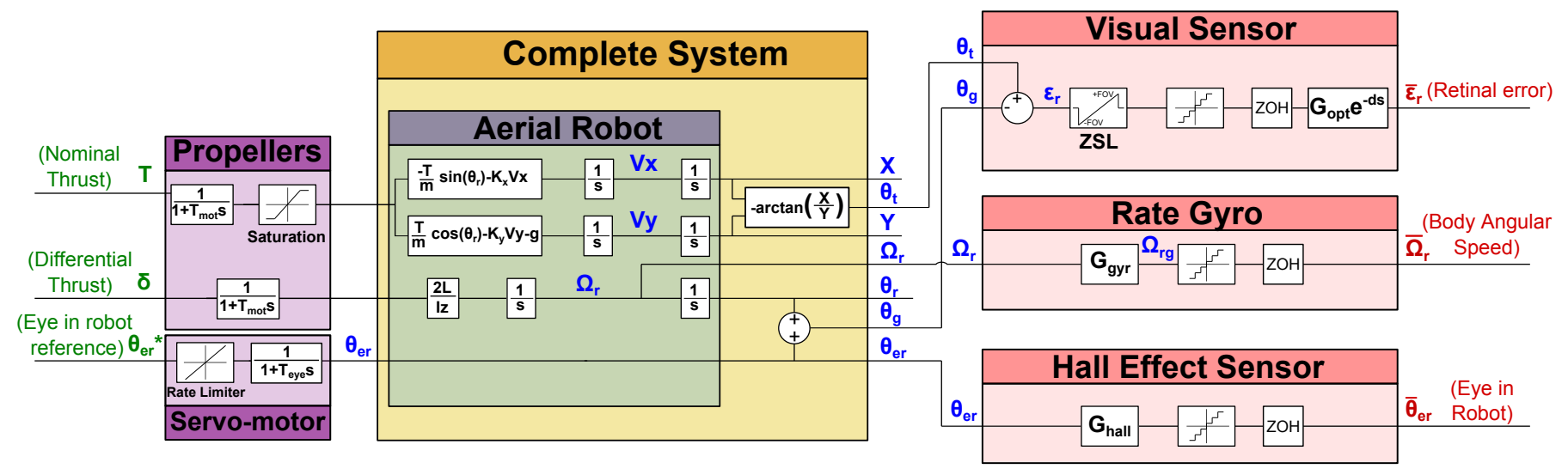

Fig. 3. Block diagramm of the complete system. Green variables represent control signal, blues correspond to the different physical variables and red correspond to different measures.

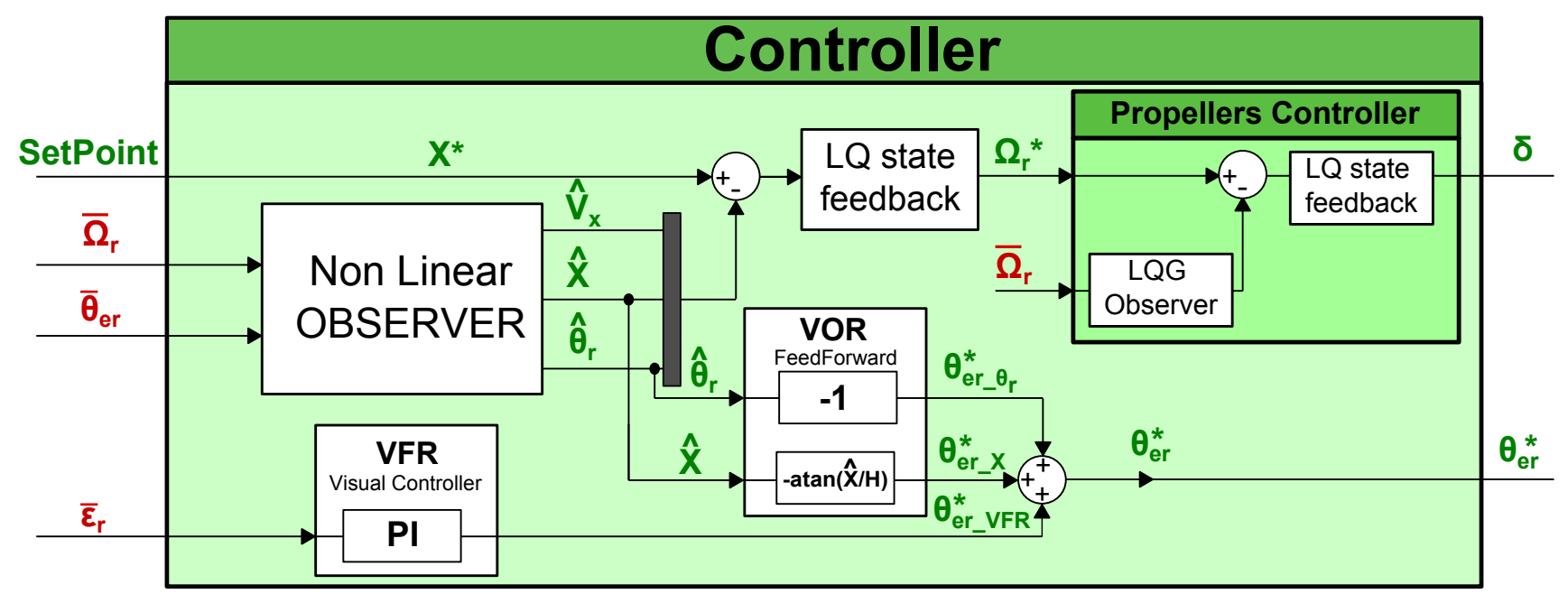

Fig. 4. Block-diagram describing the controller architecture and interconnections. The non linear observer estimates the attitude of the robot which is used by the VORs. The attitude is tracked by a state-feedback controller (LQR) and gives speed rotation set-points. Speed rotation is tracked by an LQG state-feedback controller, taking care of rate-gyro dynamic. Measured signals are in red and control signals are in green.

Where $\Omega_{r}$ is the actual rotation speed, $\Delta_{g}$ is the rate gyro bias, and $\mu$ is an unknown noise (supposed centred).

Remark 1: In system (2), for the estimation of $\theta_{r}$, it is known from (3) that the actual rotation speed is $\Omega_{r}=\bar{\Omega}_{r}-\Delta_{g}-\mu$. So the term $\bar{\Omega}_{r}-\hat{\Delta}_{g}$ is just the "model" term allowing to take care of rate gyro bias and $L_{3}\left(\bar{\theta}_{e r}-\hat{\theta}_{e r}\right)$ is the innovation term. As supposed in (4), $\hat{\hat{\Delta}}_{g}$ contains only an innovation term.

\section{Eye controller}

The control of the eyes orientation allows to keep the gaze locked onto the target placed onto the ground. In our bioinspired approach, the hovering control strategy consists of merging three complementary oculomotor reflexes :

- A rotational vestibulo ocular reflex, called rVOR, yielding at its out the signal $\theta_{e r_{-} \theta_{r}}^{\star}$ which is simply equal to the opposite of the estimated roll angle $\hat{\theta}_{r}$

- A translational vestibulo ocular reflex, called tVOR, which relies on the estimation of the robots linear position $\hat{X}$, assuming the altitude of the robot is known. This reflex compensates for any translation disturbance applied to the robots body by yielding the output signal $\theta_{e r \_\mathbb{X}}^{\star}$ which contributes to keep the eye locked onto the target.

- A visual fixation reflex where the visual feedback loop acts to cancel the retinal signal error $\epsilon_{r}$ by controlling the eyes orientation $\theta_{e r}$ through the control input signal $\theta_{e r_{-} V F R}^{\star}$ (see figure 5).

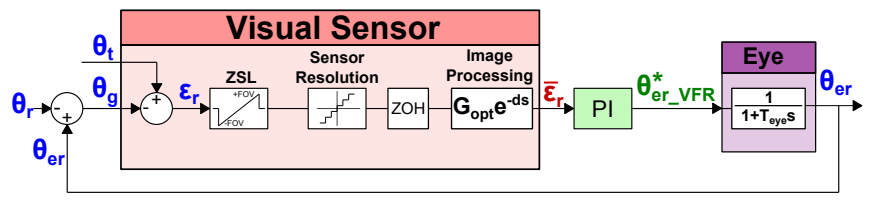

Fig. 5. Block diagram of the Visual Feedback Loop used to implement the visual fixation reflex (VFR). A PI controller acts to annul the retinal error $\epsilon_{r}$. The ZSL "Zero-Setting Limiter" is here to prevent runaway of the eye if the target is lost. $G_{o p t}$ is the static gain of the visual sensor and $d$ is a pure delay due to the image processing.

It is worth to note that the ZSL function shown in figure 5 clamps the retinal error back to zero whenever the latter tends to become higher (or lower) than a specified positive (or 
negative) level. This ZSL used in previous studies ([10], [9]) serves the same function as the limiter block used to model the inhibition of the smooth pursuit reflex whenever the position error goes beyond a fixed threshold (Citer Robinson and Stark and Young).

1) Visual Fixation Reflex control: This is the most important part of the control, which allows our robot to hover in windy conditions. This control a PI controller keeping the retinal error close to 0 . The retinal error loop control could be summarized as it is shown by figure 5 . To design the PI controller the pure delay due to image processing and the sampling is approximated by a first order Pade approximation.

The PI-controller gives an angle reference $\left(\theta_{e r_{-} V F R}^{\star}\right)$ to compensate disturbance. In this part, to prevent modelisation error, the PI-controller is designed to obtain good stability margin $\left(M_{\phi}=65, M_{M} \geq 0.5\right)$.

2) Vestibulo-Ocular Reflexes control: These reflexes allows to counteract the two kind of robot's movements, to keep the target into the FOV. The rVOR is implemented here by a feedforward, using the estimation of the roll angle $\theta_{r}$ to compensate rotation of the body. And the tVOR minimize the impact of lateral displacement onto the retinal error $\epsilon_{r}$.

3) Final eye control: To summarize, the reference angle $\theta_{e r}^{\star}$ (see figure 4) results from the contribution the three reflexes (rVOR, tVOR and VFR) as follows:

$$
\theta_{e r}^{\star}=\underbrace{\theta_{e r_{-}-\theta_{r}}^{\star}}_{r V O R}+\underbrace{\theta_{e r_{-} X}^{\star}}_{t V O R}+\underbrace{\theta_{e r_{-} V F R}^{\star}}_{V F R}
$$

So, it is easy to see in figure 6 , the contribution of the different reflexes. And in figure 7 and ?? the advantage provided by the VOR (implemented by the feedforward: $\theta_{e r_{-} \theta_{r}}^{\star}$ and $\left.\theta_{e r_{-} X}^{\star}\right)$.

To conclude, when an unknown disturbance occurs, the VFR reacts first, followed by the VORs to compensate movements engaged to reject the wind. And it is the contrary when a wished movement is accomplished: the VORs react first, and the VFR compensates model errors. The retinal error is 5 times lower with the ORs on: less than 7 while it reaches more than 35 when ORs are off. And the retinal error is vanished 6 times faster, in only 0.25 seconds with ORs activated and more than 1.5 seconds without ORs.

\section{Rotation speed controller}

The speed rotation controller enables to track the speed rotation set points $\left(\Omega_{r}^{\star}\right)$ given by the attitude controller (see III-E). To control the speed rotation it is assumed the propellers are commanded directly in thrust (see II-B).

A LQG controller-observer is chosen for tracking rather than a simple PID controller because the measure $\bar{\Omega}_{r}$ also contains the rate-gyro dynamics. This choice allows to take care of it and increase the tracking quality. So it gives the following system:

$$
\left(\begin{array}{c}
\dot{\Omega}_{r} \\
\dot{\bar{\Omega}}_{r}
\end{array}\right)=\left(\begin{array}{cc}
\frac{-2 L}{\bar{I}_{z} \tau_{m o t}} & 0 \\
\frac{1}{\tau_{\text {gyro }}} & \frac{-1}{\tau_{\text {gyro }}}
\end{array}\right)\left(\begin{array}{c}
\Omega_{r} \\
\bar{\Omega}_{r}
\end{array}\right)+\left(\begin{array}{c}
\frac{2 L}{I_{z} \tau_{m o t}} \\
0
\end{array}\right) \delta^{\star}
$$
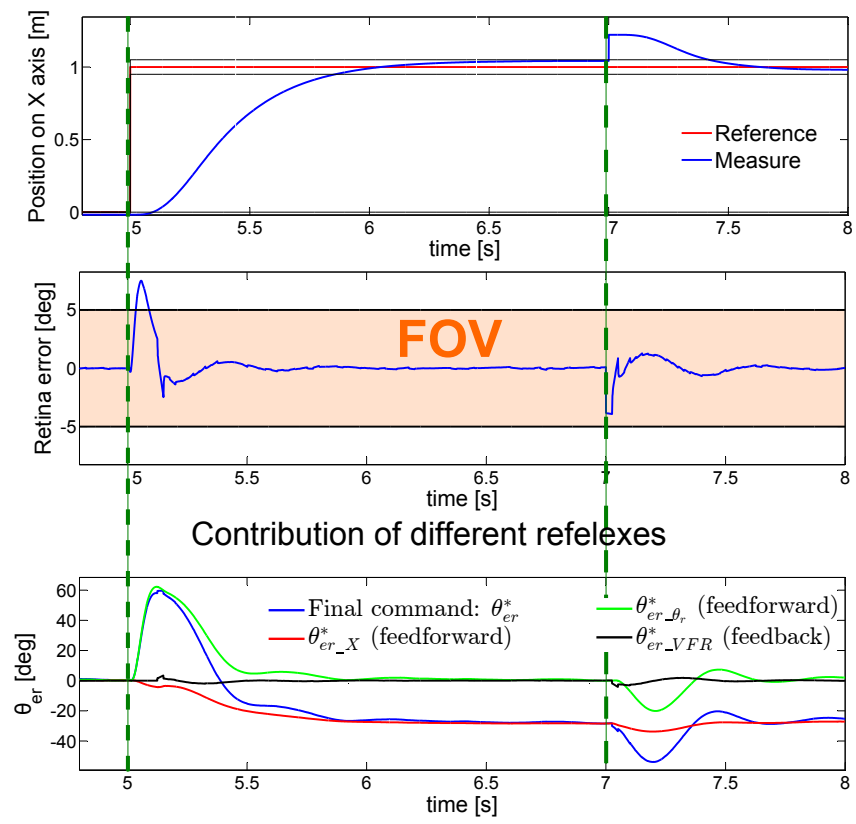

Fig. 6. Different reflexes for different situations. When a voluntary displacement appears (here the robot moves 1 meter from the target at time $t=5 s$ ), the VORs react first, and generates a rotation to compensate robot movements (the movements are estimated thanks to the observer and the control $\Omega_{r}^{\star}$ ). Then, during the displacement, the VFR appears to compensate model errors of the observer, and keep the target in FOV. Contrarily, when a lateral perturbation looms (at time $t=7 \mathrm{~s}$ ), the optic controller is the first to react, and generates a rotation to keep the target into the FOV. And only after few milliseconds, the VORs appear (when the robot begins to move to counteract the displacement generated by the disturbance).

Where $\Omega_{r}$ is the actual roll rotation speed, $\bar{\Omega}_{r}$ is the rotation speed measured by the rate-gyro.

Then an integral effect is added to reject static error in the $\Omega_{r}$ pursuit. Finally, the closed loop time response for $\Omega_{r}$ is less than $20 \mathrm{~ms}$, and the command noise is less than $2 \%$.

\section{E. Position and attitude controller}

The position and attitude controller is implemented by a pseudo LQG controller with the non linear observer. The dynamic of the speed rotation loop, previously described (see III-D) is assumed to be very fast, and could be neglected. So the control to track the position reference (on $X$ axis) and stabilize the attitude is the speed rotation of the robot $\left(\Omega_{r}\right)$. This control signals is then obtained by a simple LQG estimated-state-feedback which the estimated states $\left(V_{x}, X\right.$ and $\theta_{r}$ ) are provided by the non-linear observer.

To obtain the LQG state feedback the system (1) is linearised around the origin with the equilibrium input $\Omega_{r e q}=0$. To cancel the steady state error, an integral effect is added on the state $X$.

\section{F. Control strategy for non decoupled eye system}

The control strategy applied to the non decoupled eye robot is exactly the same than for the decoupled eye robot. The difference being that the eye is fixed (see remark below), so the rVOR, tVOR and VFR are disabled. It is assumed that the measure $\bar{\theta}_{e r}$ is directly replaced by $Z S L\left(\epsilon_{r}\right)$ (retinal 


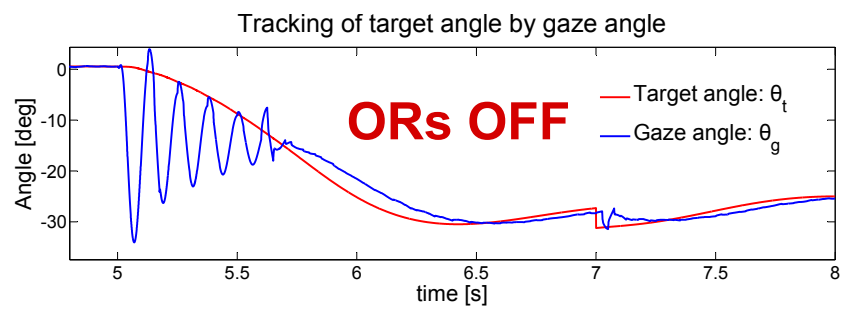

Retinal error

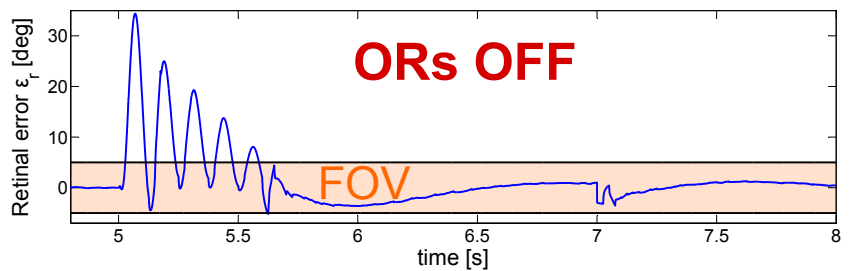

Tracking of target angle by gaze angle
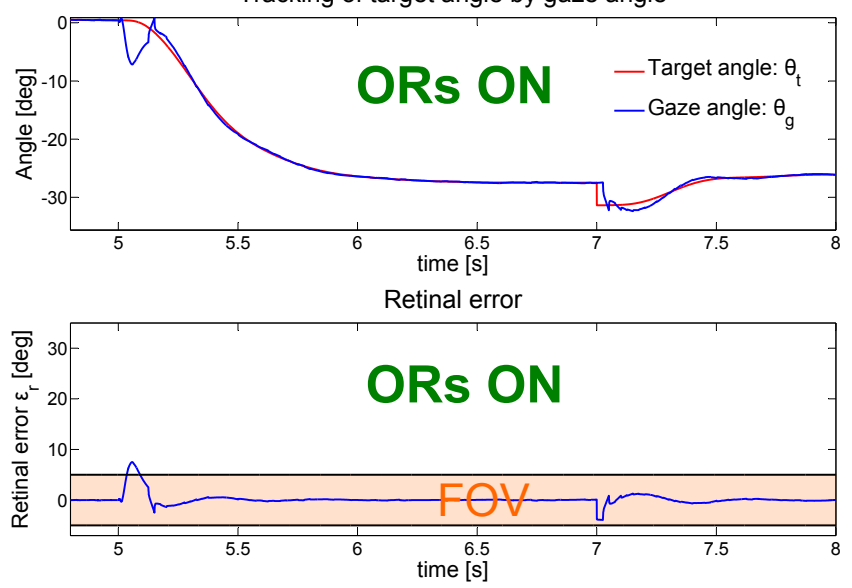

Fig. 7. When the ORs are off, the retinal error reaches more than 35 , and the eye have a lot of difficulties to lock the target because the robot movements are too quick. Luckily, when the robot come back to a roll angle close to 0 , the eye can locked the target. Note the role of the ZSL which is here very important to avoid run away of the eye. Contrarily, when the ORs are $\mathrm{ON}$, the retinal error keeps less than 7, and the target comes back to the FOV in less than $0.2 \mathrm{~s}$. The ORs allow to recover efficiency the target, even if asked movements are fast.

error and the anti run away), where:

$$
Z S L: \quad \mathbb{R} \rightarrow \mathbb{R}, \epsilon_{r} \mapsto\left\{\begin{array}{lll}
\epsilon_{r} & \text { if } & \epsilon_{r} \leqslant F O V \\
0 & \text { if } & \epsilon_{r}>F O V
\end{array}\right.
$$

So the observer is fed by $Z S L\left(\epsilon_{r}\right)$ which corresponds to the angle $\theta_{t}+\theta_{r}$, while the target is in the FOV.

Remark 2: As for the decoupled eye system, the different angles for the non decoupled eye system are linked by equation ?? with $\theta_{e r}=0$.

\section{SIMULATIONS \& COMPARISON OF BOTH BEHAVIOUR}

\section{A. Simulations conditions}

For simulations, sensors have the characterization described in appendix by tabular II. Conditions have chosen to be as close as possible to the real world. So noises and default are implemented. All parameters used for the control are voluntary worse initialised and estimated, it is mean that all parameters differs by $10 \%$ compared to the model parameters.

\section{B. Comparison of performances for the two systems}

In figure 8 and 9 , is applied to both systems, a same lateral disturbance and roll disturbance. So, a lateral disturbance, involving a half FOV displacement, is applied at time $t=$ $2.5 \mathrm{~s}$ and a roll impulse wind is applied at $t=13 \mathrm{~s}$.

1) Translation disturbances: Even if the same lateral disturbances is applied to both systems, it is easy to remark that the D-EYE system present a faster rejection in both cases. As it is shown in figure (8), the F-EYE system can not adopt big roll angle because of it necessarily implies a retinal error increase. Indeed it is visible in figure 9 that the limit of FOV is reached, for F-EYE system, even if the roll angle is small.

In conclusion, the decoupled eye allows to be more responsive to lateral disturbances without taking the risk to loose the target from FOV.

2) Rotation disturbances: In the case of the roll disturbance, the advantage of the decoupled eye system is more impressive. While the disturbance implies a big roll angle movement and a big retinal error, for no-decoupled eye system. The fast dynamic of the eye, provides to the decoupled eye system, the ability to counteract immediately the roll disturbance (retinal error and roll movements are very small.)

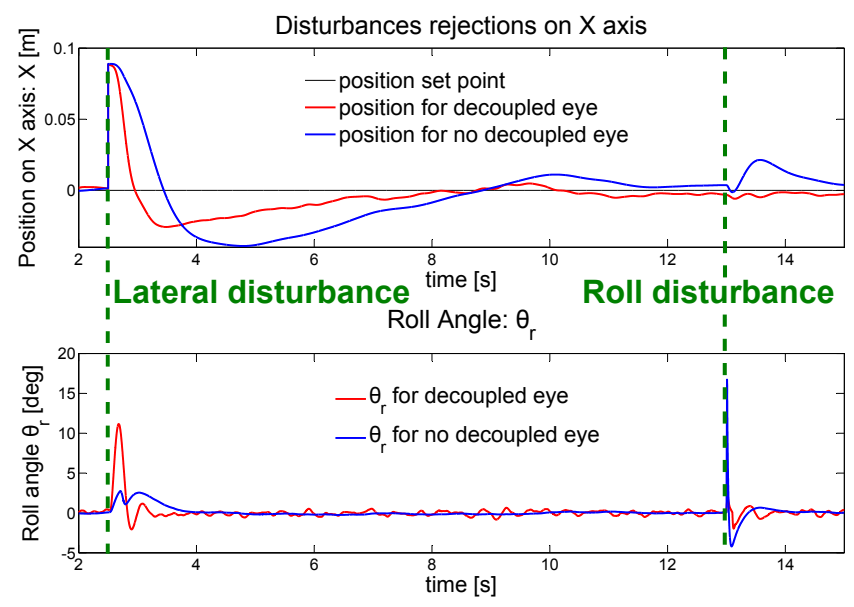

Fig. 8. Evolution of $\mathrm{X}$ position for the same lateral and roll disturbances. At the top, it is shown the evolution of the position $\mathrm{X}$, and the evolution of roll angle at the bottom.

To conclude, the decoupled eye provides a rejection, for lateral disturbances, 1.5 times faster than a classical visual robot. And a roll disturbance has induces practically any lateral displacement and less than 3 roll angle for the biomimetic robot, while the classical robot undergoes a 3 centimetres displacement and more than 15 roll angle.

\section{DISCUSSION OF A DECOUPLED EYE ADVANTAGES}

Here are balanced advantages and disadvantages to have a decoupled eye rather than no decoupled eye with a 


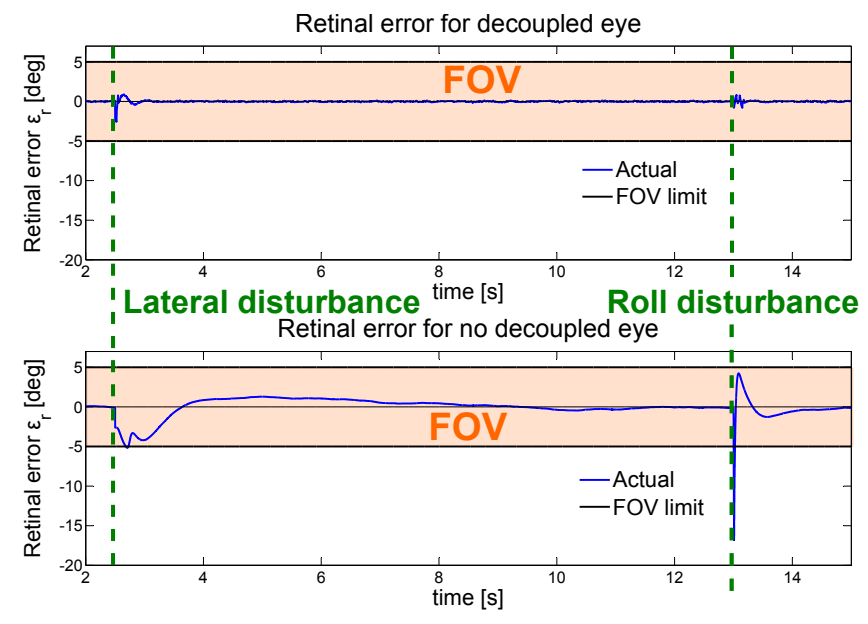

Fig. 9. Evolution of the retinal error for the two systems for the two disturbances. Retinal error for the decoupled eye system at the top and for the no-decoupled eye system at the bottom.

large FOV. The first disadvantage is naturally the additional electro-mechanical complexity introduced by the new degree of freedom. As the same, we supposed that the image treatment is not too long. Here we summarize some advantages to have a decoupled eye rather than a fixed eye with large FOV:

- Better peripheral acuity: Technically, it is difficult to obtain a sensor with a large FOV and a good peripheral vision. The peripheral image is often blurred, and worse mainly if the robot is moving. So the measured error between the target direction and the gaze $\epsilon_{r}$ would be less accurate in the periphery. A thin FOV, yields a better measurement for the retinal error.

- Reduce rotational optic flow: In the same, a decoupled eye allows to significantly reduce the rotational optic flow. And, as explained in [11], the rotational optical flow gives any informations of attitude contrary to the translatory optic flow, dominating with a decoupled eye. Then, the decoupled eye provides better informations for attitude estimation.

- Faster disturbance rejection: The decoupled eye system, enables more stiff roll angle to counteract sudden, untoward disturbances, without loosing the target from FOV. Indeed, for a same lateral disturbance, the decoupled eye system rejects 10 times faster the retinal error and 1.5 times faster the lateral offset.

- Lower calculation cost: A small FOV implies reduced image treatment.

\section{CONCLUSION}

In this paper, a new way to stabilize an aerial robot and reject wind disturbances during hovering, thanks to a decoupled eye system, is developed. This decoupled eye allows to be more efficient to reject sudden untoward disturbances thanks to occulomotor reflexes (rVOR, tVOR and VFR) possible because of the low eye's inertia. These reflexes are inspired by the insects behaviour, and provide to the robot the ability to counteract more efficiency sudden untoward disturbances. In spite of have a small field of view renders more difficult the tracking of a target, the new degree of freedom provided by the neck compensate largely this handicap. Indeed, the fast dynamics of the eye, allowing in a first hand, to keep the target in FOV thanks to ORs. And in the other hand, to provide (thanks to the proprioceptive angle sensor) a measurement of the eye-in-robot which enables to unbias the rate-gyro while estimate the current attitude and position. In this way, a new unbias approach for rate gyro is proposed, using the visual loop to estimate rate-gyro drift.

The success of the presented method is possible because the eye dynamics are very fast in comparison with the robots body dynamics. The proprioceptive sensor, providing the eye-in-robot measurement $\bar{\theta}_{e r}$, is fundamental, because it enables to estimates the attitude and position of the robot in spite of the decoupling between the eye and the body.

In a future work, this methodology will be implemented in an autonomous hovering robot, equipped with a very light and compact piezo motor to actuate the eye. And an expansion to a total autonomous aerial quadri-rotor with 6 degrees of freedom will be considered. In order to control the robot only with the measure of its gaze orientation, and the rate-gyro's datas, while rejecting sharply wind disturbances.

\section{APPENDIX:SENSORS AND ACTUATORS}

The robot is equipped with two motors and propellers allowing it to control roll angle and consequently its position on $\mathrm{X}$ axis. The height of the robot is fixed, it is mean that the robot evolves along an horizontal rail without friction. And the eye system is actuated by a servo motor allowing it to control the gaze.

A summary of the different sensors characteristics is given in the table II.

\section{ACKNOWLEDGMENT}

The authors would like to thank Robert Mahony, who have imagined with Stephane Viollet this system.

\section{REFERENCES}

[1] D. Gurdan, J. Stumpf, M. Achtelik, K.-M. Doth, G. Hirzinger, and D. Rus, "Energy-efficient autonomous four-rotor flying robot controlled at $1 \mathrm{khz}$," in Robotics and Automation, 2007 IEEE International Conference on, april 2007, pp. $361-366$.

[2] S. P. Jinhyun Kim, Min-Sung Kang, "Accurate modeling and robust hovering control for a quad-rotor vtol aircraft," Journal of Intelligent Robotic Systems, vol. 57, pp. 9-26, 2010.

[3] T. K. Ryosuke Mori, Kenichi Hirata, "Vision-based guidance control of a small-scale unmanned helicopter," in Conference on Intelligent Robots and Systems, Oct 29 - Nov 22007.

[4] T. Zhang, Y. Kang, M. Achtelik, K. Kuhnlenz, and M. Buss, "Autonomous hovering of a vision/imu guided quadrotor," in International Conference on Mechatronics and Automation, 2009.

[5] A. Z. Karl E.Wenzel, Paul Rosset, "Low-cost visual tracking of a landing place and hovering flight control with a microcontroller," in Selected papers from the 2nd international Symposium on UAV, 2009, pp. 297-311.

[6] E. Rondon, L.-R. Garcia-Carrillo, and I. Fantoni, "Vision-based altitude, position and speed regulation of a quadrotor rotorcraft," in Conference on Intelligent Robots and Systems, Oct 18-22 2010. 


\begin{tabular}{|c|c|c|c|}
\hline Parameter & Description & Value & Unit \\
\hline$L$ & $\begin{array}{l}\text { Half span of } \\
\text { robot }\end{array}$ & 0.15 & $m$ \\
\hline$m$ & Mass of robot & 0.1 & $k g$ \\
\hline$I_{z}$ & $\begin{array}{l}\text { Inertia } \\
\text { momentum }\end{array}$ & $2.0 \times 10^{-5}$ & $\mathrm{~kg} \cdot \mathrm{m}^{2}$ \\
\hline$K_{v x}$ & $\begin{array}{l}\text { Flapping } \\
\text { coefficient }\end{array}$ & 0.5 & N.s.m $m^{-1}$ \\
\hline$F O V$ & $\begin{array}{l}\text { Field of View } \\
\text { Image }\end{array}$ & 5 & \\
\hline$d$ & $\begin{array}{l}\text { treatment } \\
\text { delay }\end{array}$ & 10 & $m s$ \\
\hline$G_{o p t}$ & $\begin{array}{l}\text { Optic sensor's } \\
\text { gain }\end{array}$ & 1 & - \\
\hline$\tau_{\text {hall }}$ & $\begin{array}{l}\text { Angle sensor's } \\
\text { time constant }\end{array}$ & 1 & $m s$ \\
\hline$\tau_{g y r}$ & $\begin{array}{l}\text { Rate gyro's } \\
\text { time constant }\end{array}$ & 4.3 & $m s$ \\
\hline$\tau_{m o t}$ & $\begin{array}{l}\text { Propeller's } \\
\text { time constant } \\
\text { Eye }\end{array}$ & 20 & $m s$ \\
\hline$\tau_{\text {eye }}$ & $\begin{array}{l}\text { mechanism } \\
\text { time constant }\end{array}$ & 1 & $m s$ \\
\hline
\end{tabular}

TABLE I

SIMULATIONS PARAMETERS.

\begin{tabular}{|l|c|c|c|}
\hline \multicolumn{5}{|c|}{ Sensors } \\
\hline & Visual sensor & Angle sensor & Rate gyro \\
\hline $\begin{array}{l}\text { Transfer } \\
\text { function }\end{array}$ & $G_{\text {opt }}(s)=G_{\text {opt }} e^{-d s}$ & $G_{\text {hall }}(s)=\frac{1}{1+\tau_{\text {hall }}}$ & $G_{\text {gyr }}(s)=\frac{1}{1+\tau_{\text {gyr }}}$ \\
\hline Resolution & 0.1[] & 0.1[] & $1\left[. s^{-1}\right]$ \\
\hline $\begin{array}{l}\text { Sample } \\
\text { frequency }\end{array}$ & $40[\mathrm{~Hz}]$ & $1[\mathrm{kHz}]$ & $1[\mathrm{kHz}]$ \\
\hline $\begin{array}{l}\text { Noise } \\
\text { ampli- } \\
\text { tude }\end{array}$ & \pm 0.1[] & \pm 1[] & $\pm 5\left[. s^{-1}\right]$ \\
\hline \multicolumn{4}{|c|}{ Actuators } \\
\hline $\begin{array}{l}\text { Transfert } \\
\text { function }\end{array}$ & $G_{\text {mot }}(s)=\frac{1}{1+\tau_{\text {mot }}{ }^{s}}$ & Eye motor \\
\hline $\begin{array}{l}\text { Rate } \\
\text { limiter }\end{array}$ & - & $G_{\text {servo }}(s)=\frac{1}{1+\tau_{\text {eye }} s}$ \\
\hline
\end{tabular}

TABLE II

SENSORS AND ACTUATORS CHARACTERISTICS.

[7] D. Schafroth, C. Bermes, S. Bouabdallah, and R. Siegwart, "Modeling, system identification and robust control of a coaxial micro helicopter," Control Engineering Practice, vol. 18, no. 7, pp. 700-711, 2010.

[8] N. F. L. Kerhuel, S. Viollet, "A sighted aerial robot with fast gaze and heading stabilization," in Conference on the Intelligent Robots and Systems, Oct 29 - Nov 22007.

[9] L. Kerhuel, S. Viollet, and N. Franceschini, "Steering by gazing: An efficient biomimetic control strategy for visually guided micro aerial vehicles," Robotics, IEEE Transactions on, vol. 26, no. 2, pp. 307 -319 , april 2010.

[10] S. Viollet and N. Franceschini, "A high speed gaze control system based on the vestibulo-ocular reflex," Robotics and Autonomous Systems, vol. 50, pp. 147-161, 2005.
[11] J. Hateren and C. Schilstra, "Blowfly flight and optic flow. ii. head movements during flight," Journal of Experimental Biology, vol. 202, no. 11, p. 1491, 1999. 